\title{
Metástasis cutáneas de cáncer de recto
}

\section{Cutaneous metastases from rectal cancer}

\author{
M.J. de Miguel Valencia ${ }^{1}$, M. Fraile González ${ }^{2}$, A. Yagüe Hernando ${ }^{3}$, F. Oteiza Martínez ${ }^{1}$, \\ M.A. Ciga Lozano ${ }^{1}$, P. Armendáriz Rubio ${ }^{1}$, M. de Miguel Velasco ${ }^{1}$, H. Ortiz Hurtado ${ }^{1}$
}

\section{RESUMEN}

El carcinoma colorrectal es un tumor que con muy poca frecuencia origina metástasis cutáneas, y cuando lo hace raramente es por vía hematógena. Presentamos el caso de un varón de 55 años diagnosticado de un adenocarcinoma de recto (tercio inferior) estadio T3N2M0. Tratado inicialmente con radioquimioterapia neoadyuvante, fue intervenido ocho semanas después realizándose una amputación abdominoperineal extendida. El resultado anatomopatológico fue de adenocarcinoma mucinoso de recto, estadio ypT3bN1. Tras un postoperatorio sin complicaciones el paciente recibió tratamiento quimioterápico con capecitabina.

A los 18 meses el paciente refirió la aparición progresiva de nódulos subcutáneos en distintas localizaciones. En la tomografía computarizada de control se objetivaron múltiples imágenes sugestivas de metástasis a nivel hepático y pulmonar, así como lesiones subcutáneas. La exéresis-biopsia de uno de los nódulos subcutáneos corroboró la sospecha de metástasis de adenocarcinoma de recto. Se valoró tratamiento quimioterápico que no llegó a administrarse por el rápido deterioro del paciente que llevó al exitus.

Palabras clave. Cáncer rectal. Metástasis cutáneas.

\begin{abstract}
Colorectal carcinoma is a tumour that very infrequently gives rise to cutaneous metastases and when it does so, it is rarely by the haematogenous route. We present the case of a 55-year old male diagnosed with an adenocarcinoma of the rectum (lower third), clinical stage T3N2M0. Initially treated with neoadjuvant radiochemotherapy, he was operated on eight weeks later, with an extended abdominoperineal amputation. The anatomopathological result was mucinous adenocarcinoma of the rectum, clinical stage ypT3bN1. Following a postoperative period without complications, the patient received chemotherapeutic treatment with capecitabine.

Eighteen months later the patient reported the progressive appearance of subcutaneous nodules in different localizations. In the computerized tomography test multiple images were objectivized suggesting metastasis at the hepatic and pulmonary levels, as well as subcutaneous lesions. The biopsy-excision of one of the subcutaneous nodules corroborated the suspicion of metastasis of the adenocarcinoma of the rectum. Chemotherapy treatment was considered for the patient, which was not administered due to the rapid deterioration of the patient leading to his death.
\end{abstract}

Key words. Rectal cancer. Cutaneous metastases.
1. Unidad Clínica de Cirugía Colorrectal y Proctología. Área de Cirugía. Complejo Hospitalario de Navarra. Pamplona (España)

2. Servicio de Aparato Digestivo. Complejo Hospitalario de Navarra. Pamplona (España)

3. Servicio de Anatomía Patológica. Complejo Hospitalario de Navarra. Pamplona (España)

Recepción: 9 de enero de 2013

Aceptación provisional: 15 de febrero de 2013

Aceptación definitiva: 26 de marzo de 2013

\section{Correspondencia:}

Mario Javier de Miguel Valencia Unidad Clínica de Cirugía Colorrectal y Proctología. Área de Cirugía Complejo Hospitalario de Navarra. Edificio B $\mathrm{C} /$ Irunlarrea $\mathrm{n}^{\circ} 4$ 31008 Pamplona, Navarra mariodemiguel85@gmail.com 


\section{INTRODUCCIÓN}

En nuestro medio, el cáncer colorrectal es el segundo cáncer más frecuente en la mujer y el tercero en el hombre ${ }^{1}$. Los tumores de colon y recto pueden diseminarse por contigüidad, vía linfática, vía peritoneal y vía hematógena. A través de la sangre, el hígado es el órgano más frecuentemente afectado, estimándose que aproximadamente la mitad de los pacientes desarrollará metástasis hepáticas en algún momento de su evolución ${ }^{2}$.

La piel es un órgano raramente afectado en la diseminación de un cáncer colorrectal; las metástasis cutáneas se presentan en un porcentaje inferior al $4 \%^{3-6}$, y cuando ocurren suelen localizarse en zonas relativamente cercanas al tumor primario (cicatrices quirúrgicas por implante de células durante la cirugía), siendo la diseminación cutánea a distancia, vía hematógena, muy poco frecuente ${ }^{7}$.

Presentamos el caso de un paciente con cáncer de recto que en su evolución presentó múltiples metástasis cutáneas a distintos niveles, un evento extremadamente raro.

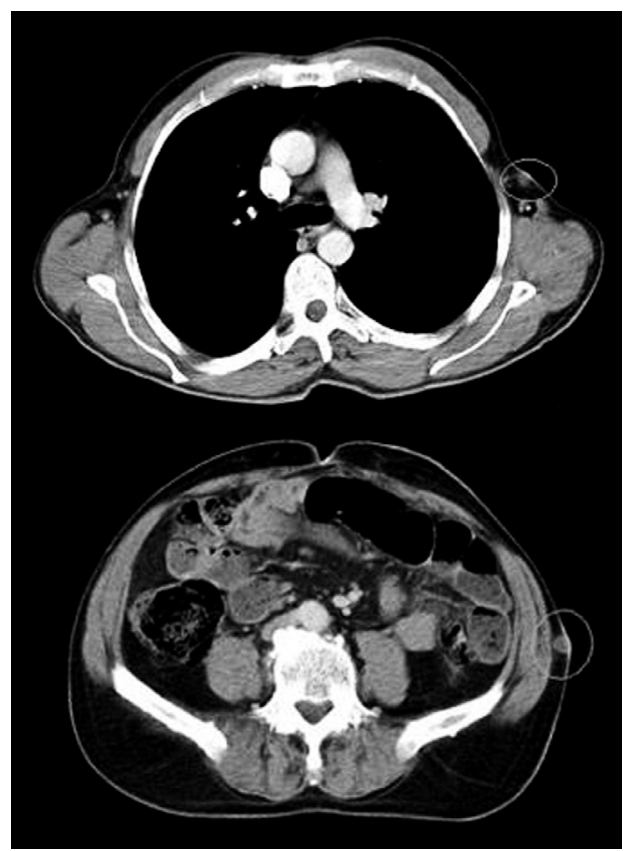

\section{CASO CLÍNICO}

Varón de 55 años, sin antecedentes de interés, estudiado por cuadro de alteración del ritmo intestinal y rectorragias, que tras endoscopia y biopsia es diagnosticado de un adenocarcinoma de tercio inferior de recto, bien diferenciado, de alto grado, con diferenciación mucinosa en menos del $50 \%$, con estadiaje clínico-radiológico (tomografía computerizada y resonancia magnética) T3 N2M0.

El paciente fue tratado inicialmente con radioquimioterapia neoadyuvante; radioterapia en esquema de ciclo largo con una dosis total de 50,4 Gy durante 5 semanas, y capecitabina vía oral a razón de $825 \mathrm{mg} / \mathrm{m}^{2} / 12 \mathrm{~h}(1650 \mathrm{mg} / 12 \mathrm{~h})$, cada 15 días.

Ocho semanas después de finalizar el tratamiento fue intervenido quirúrgicamente realizándose una amputación abdominoperineal extendida. El resultado anatomopatológico de la pieza quirúrgica fue de adenocarcinoma mucinoso de recto, a $4,5 \mathrm{~cm}$ de margen anal, cuya infiltración sobrepasaba $2 \mathrm{~mm}$ la muscular propia, con bordes quirúrgicos proximal y distal sin tumor, y margen de resección circular a $20 \mathrm{~mm}$ del tumor y a $2 \mathrm{~mm}$ de una adenopatía metastatizada, con un total de 3 ganglios positivos de los 10 disecados. La escisión mesorrectal (en el plano del mesorrecto) y de canal anal (en el plano de los elevadores) fueron satisfactorias (ypT3bN1).
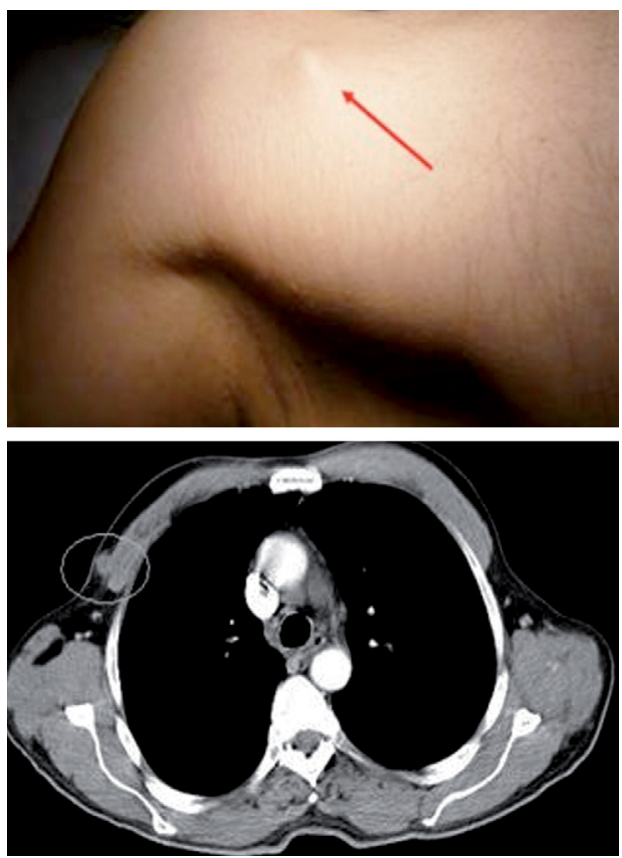
El postoperatorio del paciente cursó sin complicaciones, y posteriormente recibió 6 ciclos de quimioterapia con capecitabina vía oral a razón de $2.000 \mathrm{mg} / 12 \mathrm{~h}$ cada 21 días.

En su evolución fue controlado por los servicios de Oncología y Cirugía General, con TC, colonoscopia y marcadores tumorales normales al año de la intervención. En su revisión rutinaria a los 18 meses de la intervención, el paciente refiere la aparición progresiva de nódulos subcutáneos faciales en zona frontal, en axila izquierda, pectoral derecho, flanco izquierdo y extremidades inferiores. A la exploración física se corrobora la existencia de nódulos subcutáneos de entre 0,5 y $1 \mathrm{~cm}$ en las localizaciones descritas, de consistencia elástica y rodaderos. Se realizó TC tóracoabdominal donde se objetivaron múltiples imágenes sugestivas de metástasis a nivel hepático y pulmonar, así como lesiones subcutáneas localizadas en el área pectoral derecha área axilar izquierda, pared abdominal anterior, flanco izquierdo y áreas glúteas (Figs. 1 y 2).

Se realizó exéresis-biopsia de uno de los nódulos subcutáneos localizado en región pectoral derecha (Fig. 2) con resultado anatomopatológico de metástasis subcutánea de adenocarcinoma bien diferenciado de recto. Ante el diagnóstico de recidiva tumoral diseminada se valoró nuevamente tratamiento quimioterápico que el paciente no llegó a recibir por el rápido deterioro de su estado general que le llevó al exitus.

\section{DISCUSIÓN}

Las bases biológicas de la diseminación metastásica tumoral son complejas y no se conocen completamente ${ }^{3}$. A pesar de que el tejido blando constituye aproximadamente un 55\% de nuestra masa corporal, es raro que existan metástasis en estas áreas, siendo el diagnóstico histopatológico más frecuente el adenocarcinoma ${ }^{8}$. La prevalencia de metástasis cutáneas de tumores viscerales supone un $2 \%$ de todos los tumores de piel y con frecuencia aparecen junto con metástasis en otros órganos ${ }^{9}$. De acuerdo con las series de casos encontradas en la literatura, son el melanoma, el cáncer de mama y los cánceres del tracto respiratorio superior los que con mayor frecuencia metastatizan en la piel. Por el contrario, el cáncer colorrectal raramente origina metástasis cutáneas ${ }^{3,9-11}$.
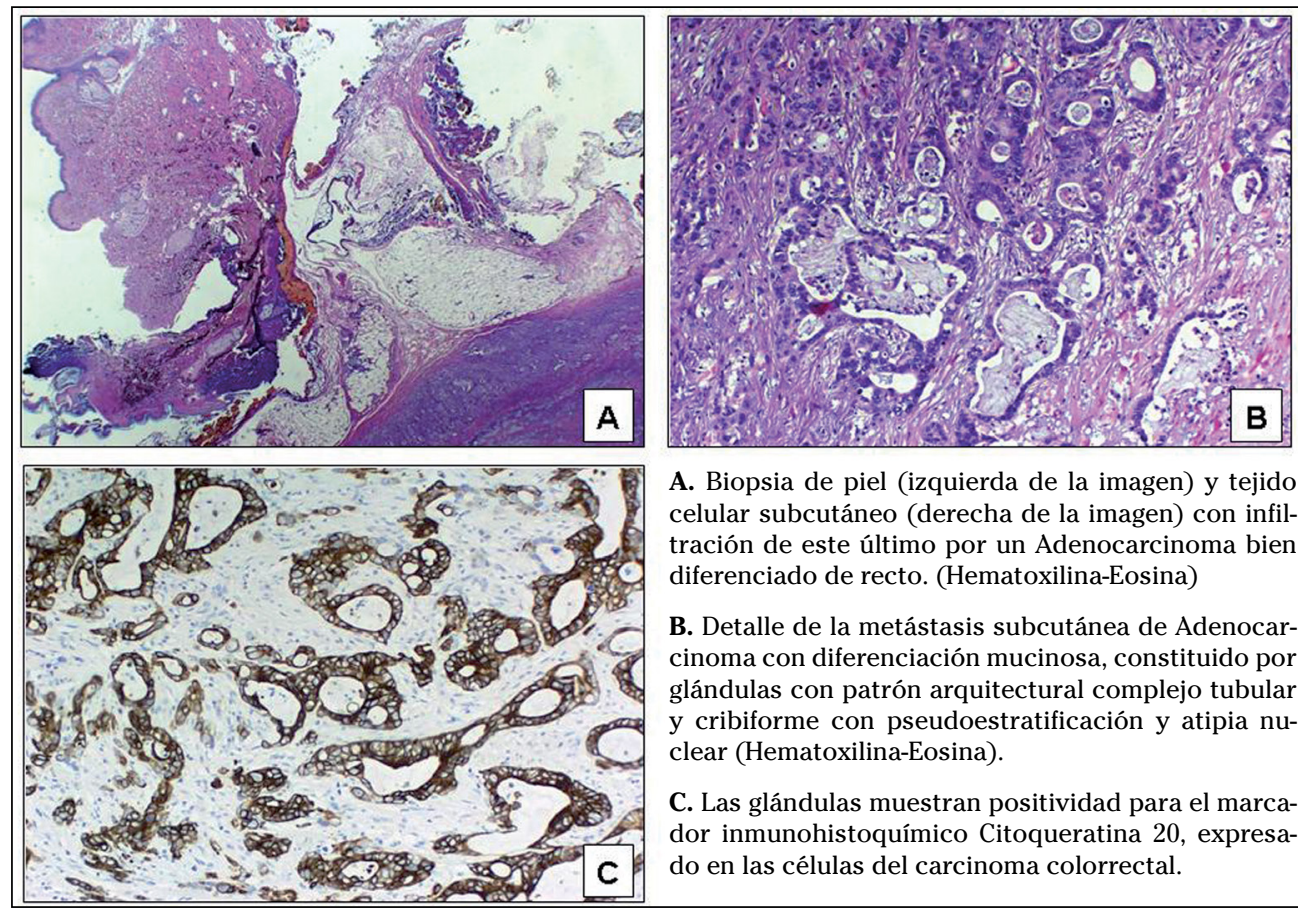

A. Biopsia de piel (izquierda de la imagen) y tejido celular subcutáneo (derecha de la imagen) con infiltración de este último por un Adenocarcinoma bien diferenciado de recto. (Hematoxilina-Eosina)

B. Detalle de la metástasis subcutánea de Adenocarcinoma con diferenciación mucinosa, constituido por glándulas con patrón arquitectural complejo tubular y cribiforme con pseudoestratificación y atipia nuclear (Hematoxilina-Eosina).

C. Las glándulas muestran positividad para el marcador inmunohistoquímico Citoqueratina 20, expresado en las células del carcinoma colorrectal. 
En el cáncer colorrectal es más frecuente encontrar células tumorales en piel debido a extensión directa o implantación local durante la cirugía en las zonas de la incisión quirúrgica (región periumbilical, perianal o sitio de implantación de trócares de laparoscopia), si bien la incidencia de este evento ha disminuido notablemente con la mejora de las técnicas quirúrgicas.

Las metástasis cutáneas de cáncer colorrectal no presentan unas características específicas; por lo general se trata de nódulos subcutáneos o intradérmicos de 1-2 cm de diámetro, inicialmente asintomáticos, sin cambios epidérmicos, y pueden confundirse con quistes, lipomas y granulomas. En ocasiones pueden presentar un crecimiento rápido, ulcerarse y producir celulitis ${ }^{4}$. Cuando se sospecha la afectación cutánea puede emplearse la biopsia o la (punción-aspiración con aguja fina) PAAF como método de obtención de tejido para el diagnóstico histopatológico, pudiendo ser este último más rápido y eficiente ${ }^{12}$. En nuestro caso se realizó exéresis de un nódulo subcutáneo en región pectoral.

El patrón histopatológico que se observa en las metástasis de adenocarcinomas viscerales consiste en la presencia de estructuras glandulares, tapizadas por un epitelio atípico y que no corresponde a ninguna estructura anexial cutánea. En la biopsia cutánea de nuestro caso, se observó la epidermis y la dermis masivamente infiltradas por un adenocarcinoma bien diferenciado (Fig. 3 A), constituido por glándulas que mostraban un patrón arquitectural complejo tubular y cribiforme, con pseudoestratificación y atipia nuclear con presencia de mitosis (Fig. 3 B).

Las metástasis cutáneas se deben distinguir histológicamente de un tumor cutáneo primario, y no siempre es fácil ${ }^{11}$, para ello existen gran variedad de paneles inmunohistoquímicos, paneles de anticuerpos y test genéticos que pueden ser de utilidad $^{7,11}$. En nuestro caso, las glándulas mostraban positividad para el marcador inmunohistoquímico de enterocitos citoqueratina 20 (Fig. $3 \mathrm{C}$ ) y para el marcador inmunohistoquímico de diferenciación colorrectal CDX-2.
Considerando el hallazgo como un dato de estadio avanzado de la enfermedad, el tratamiento es generalmente paliativo ${ }^{9}$. Con esta finalidad, si las lesiones son sintomáticas puede considerarse como primera elección la escisión local de la lesión o la radioterapia. Otras opciones son braquiterapia, quimioterapia sistémica o intralesional, inmunoterapia, electrocoagulación y electrovaporización ${ }^{3,9}$. Recientemente se ha publicado la efectividad de la electroquimioterapia con bleomicina en el control local y alivio sintomático de la enfermedad metastásica cutánea ${ }^{13}$. No obstante, son pocos los datos existentes actualmente sobre la efectividad de las distintas modalidades de tratamiento.

Finalmente, debemos incidir en el hecho de que la aparición de metástasis cutáneas de cáncer colorrectal representa en la mayoría de los casos un estadio muy avanzado de la enfermedad ${ }^{14}$, y que por tanto el tratamiento es habitualmente sólo paliativo.

\section{BIBLIOGRAFÍA}

1. Ardanaz E, Moreno Iribas C, Pérez de Rada ME, Ezponda C, Agorreta A et al. Incidencia y mortalidad por cáncer en Navarra 1993-1997. An Sist Sanit Navar 2001; 24: 339-362.

2. Herrera J, Balén E, ZaZpe C, Lera JM. Estado actual del tratamiento quirúrgico de las metástasis hepáticas de origen colorrectal: una visión práctica. An Sist Sanit Navar 2002; 25 : 317-325.

3. LoOKIngBill DP, Spangler N, Helm KF. Cutaneous metastases in patients with metastatic carcinoma: a retrospective study of 4020 patients. J Am Acad Dermatol 1993; 29: 228-236.

4. SARID D, Wigler N, GutKin Z, Merimsky O, LeiderTrejo L, Ron IG. Cutaneous and subcutaneous metastases of rectal cancer. Int J Clin Oncol 2004; 9: 202-205.

5. Schwartz RA. Cutaneous metastatic disease. J Am Acad Dermatol 1995; 33: 161-182.

6. Gmitter TL, Dhawan SS, Phillips MG, Wiszniak J. Cutaneous metastases of colonic adenocarcinoma. Cutis 1990; 46: 66-68.

7. Nashan D, Meiss F, Braun-Falco M, Reichenberger S. Cutaneous metastases from internal malignancies. Dermatol Ther 2010; 23: 567-580.

8. Plaza JA, Pérez-Montiel D, Mayerson J, MorriSON C, Suster S. Metastases to soft tissue: a 
review of 118 cases over a 30 -year period. Cancer 2008; 112: 193-203.

9. Nashan D, Müller ML, Braun-Falco M, ReichenBerger S, Szeimies RM, Bruckner-Tuderman L. Cutaneous metastases of visceral tumours: a review. J Cancer Res Clin Oncol 2009; 135: 1-14.

10. Hu SC, Chen GS, Wu CS, Chai CY, Chen WT, LaN CC. Rates of cutaneous metastases from different internal malignancies: experience from a Taiwanese medical center. J Am Acad Dermatol 2009; 60: 379-387.

11. FernÁNDEZ-Flores A. Cutaneous metastases: a study of 78 biopsies from 69 patients. Am J Dermatopathol 2010; 32: 222-239.
12. Bansal R, Patel T, Sarin J, Parikh B, Ohri A, TriVEDI $P$. Cutaneous and subcutaneous metastases from internal malignancies: an analysis of cases diagnosed by fine needle aspiration. Diagn Cytopathol 2011; 39: 882-887.

13. Matthiessen LW, Chalmers RL, Sainsbury DC, Veeramani S, Kessell G, Humphreys AC et al. Management of cutaneous metastases using electrochemotherapy. Acta Oncol 2011; 50: 621-629.

14. Gazoni LM, Hedrick TL, Smith PW, Friel CM, Swenson BR, ADAms JD et al. Cutaneous metastases in patients with rectal cancer: a report of six cases. Am Surg 2008; 74: 138-140. 
